\title{
Treatment-related decisional conflict in patients with depressive and anxious disorders
}

\author{
This article was published in the following Dove Press journal: \\ Patient Preference and Adherence \\ 10 June 2016 \\ Number of times this article has been viewed
}

\author{
Carlos De las Cuevas' \\ Ramsés Marrero' \\ Casimiro Cabrera ${ }^{2}$ \\ 'Department of Internal Medicine, \\ Dermatology and Psychiatry, \\ University of La Laguna, San Cristóbal \\ de La Laguna, Canary Islands, Spain; \\ ${ }^{2}$ Department of Psychiatry, Queen's \\ University, Kingston, ON, Canada
}

Purpose: To determine the level of treatment-related decisional conflict in patients with emotional disorders and to establish its relationship with sociodemographic and clinical variables.

Methods: We conducted a cross-sectional survey on a convenience sample of 321 consecutive psychiatric outpatients with emotional disorders. All patients completed self-report questionnaires assessing sociodemographic and clinical variables, patients' preference of participation in decision making, perceived decisional conflict about treatment, adherence to prescribed treatment, and satisfaction with the psychiatric care provided. Multiple correspondences analysis was used to investigate relationships of decisional conflict with the variables of interest.

Results: Approximately, two-thirds of psychiatric outpatients self-reported decisional conflict regarding their treatment. Interestingly, the presence of decisional conflict did not influence significantly patients' preferences of participation or their adherence to prescribed treatment. Patients without decisional conflict registered significantly higher satisfaction. Multiple correspondences analysis evidenced two clear profiles: patients without decisional conflict received the treatment they preferred, mainly psychotherapy or combined treatment, had been under psychiatric treatment for longer than 5 years, and self-reported high satisfaction with health care received; on the other hand, patients with decisional conflict did not receive the treatment they preferred, were treated with pharmacotherapy alone for a period of time between 1 and 5 years, and self-reported medium satisfaction with received health care.

Conclusion: The high level of decisional conflict found in patients with depression and anxiety attending a secondary care service could be an important driving force when personalizing and tailoring information and teaching skills to patients about their illnesses and their treatments.

Keywords: decisional conflict, psychiatric patient involvement, treatment adherence, patient satisfaction

\section{Introduction}

If psychiatry were an accurate science-based medical specialty, there would be one appropriate answer for each mental health problem and the patients' preferences about treatments would be irrelevant to what is "right". But, psychiatry remains just an uncertain science with many clinical situations in which more than one reasonable possibility of intervention is available with no evidence that any of the options is better than another. ${ }^{1}$

In this context, and despite the fact that patient participation in decision making meets the ethical principle of patient autonomy and the legal requirement of informed consent $^{2}$ and has also been associated to better patients' outcome measures, ${ }^{3}$ current literature on the topic reports significant variability in how much patients want to participate in this process. ${ }^{4-6}$ Moreover, making the right medical choices is harder than ever since patients are overwhelmed by information from all sides: their doctors'
Correspondence: Carlos De las Cuevas Department of Internal Medicine, Dermatology and Psychiatry, School of Health Sciences, Medicine Section, University of La Laguna, Campus de Ofra, 3807I San Cristóbal de La Laguna,

Canary Islands, Spain

Tel +34 609 52I 405

Email cdelascuevas@gmail.com 
recommendations, the dissenting opinions of the experts, the presence of confusing statistics, conflicting media reports, the advice of friends, claims on the Internet, and a never-ending stream of drug company advertisements. ${ }^{7}$

Depression and anxiety disorders are among the most common illnesses in the community of Western countries with estimated lifetime prevalence rates of up to $29 \%$ for anxiety disorders and 19\% for depressive disorders. ${ }^{8}$ Patients with depression often have significant symptoms of anxiety disorders, ${ }^{9}$ and those with anxiety disorders often also have depression. ${ }^{10}$ Furthermore, both psychiatric disorders may occur together, meeting criteria for both. ${ }^{11}$ Current empirical evidence relating to biological and psychological vulnerabilities, comorbidity, latent structure, cognitive and behavioral maintaining factors, and treatment outcome suggests that commonalities across anxiety and depressive disorders are greater than differences. ${ }^{12,13}$ In light of these findings, patient participation in decision making represents a key factor that needs to be addressed at the time of improving the adherence to treatment and the clinical outcome of emotional disorders, ${ }^{14}$ since without treatment, they can last longer and recur more often.

A recent systematic overview on the efficacy of pharmacotherapies and psychotherapies for major psychiatric disorders ${ }^{15}$ has shown that although many pharmacotherapies and psychotherapies are effective, there is considerable room for improvement because of the multiple differences in the methods used in pharmacotherapy and psychotherapy trials. In the field of depressive and anxiety disorders, a recent meta-analysis of direct comparisons has concluded that pharmacotherapy and psychotherapy have comparable effects in several depressive and anxiety disorders, although this is not true for all disorders, especially not for obsessivecompulsive disorder and possibly dysthymia. ${ }^{16}$

In patients with depression, the available evidence suggests no difference in treatment effects of second-generation antidepressants and cognitive behavioral therapies, either alone or in combination. ${ }^{17}$ According to Amick et al, ${ }^{17}$ given that patients may have personal preferences for one first-line treatment over the other, both treatments should be made accessible, either alone or in combination, to primary care patients with major depressive disorder, and the most efficient option for the treatment of first-episode major depressive disorder, therefore, may simply be to follow the patient's preference.

So, every day in clinical practice psychiatric patients are faced with making choices about alternative actions and have to deal with the uncertainty about which course of action to take; when choice among competing actions involves risk, loss, regret, or challenge to personal life values, this is termed decisional conflict. ${ }^{18}$

The aim of the present study was to determine the prevalence and characteristics of treatment-related decisional conflict perceived by psychiatric outpatients with emotional disorders; to examine its influence on the desired roles that these outpatients want to play about their treatment; and to establish the relationship of decisional conflict with satisfaction, with received care, and with adherence to prescribed treatment. The following hypotheses were generated: 1) preferences for being involved in psychiatric treatment decisions are related to the presence of decisional conflict; 2) lower decisional conflict positively influences satisfaction with the psychiatrist and adherence to prescribed treatment; and 3) congruence between received and preferred treatment influences patient's satisfaction and adherence to treatment.

\section{Materials and methods Sample}

From July 2015 to November 2015, 450 consecutive psychiatric outpatients seen in two Community Mental Health Centers on Tenerife Island (Canary Islands, Spain) were invited to participate in the study. Patients were eligible for inclusion in the study if they were aged 18 years and over and were diagnosed by their psychiatrists with psychiatric disorders using the International Classification of Diseases, tenth edition (ICD-10) ${ }^{19}$ coded as F32-33 (depressive episode and recurrent depressive disorder) and F40-48 (panic disorder, generalized anxiety disorder, adjustment disorder, and obsessive-compulsive disorder). Each participant received a full explanation of the study, after which, all participants signed an informed consent document. This study was approved by the Ethics Committee for Clinical Research of the University Hospital Nuestra Señora de la Candelaria. Each participant then filled out a brief sociodemographic survey and the rest of the questionnaires.

\section{Measures}

\section{Sociodemographic characteristics and clinical variables}

The variables such as age, sex, educational level (primary studies, secondary studies, and university degree), diagnoses, time under psychiatric treatment, and current and preferred psychiatric treatment (psychotherapy alone, pharmacotherapy alone, and psychotherapy combined with pharmacotherapy) were registered.

\section{Instruments}

"Decisional conflict" was measured using the SURE test (Sure of myself; Understand information; Risk-benefit 
ratio; Encouragement). ${ }^{20}$ This is a four-item self-reported scale with two response categories (yes/no) that measures personal perceptions of uncertainty in choosing options, modifiable factors contributing to uncertainty such as feeling uninformed, unclear about personal values, and unsupported in decision making. Responses are scored as yes (score $=1$ ) or no (score $=0$ ). Scores of less than 3 indicate decisional conflict. The internal reliability of SURE test was moderate (Cronbach's $\alpha=0.65){ }^{20}$

The Control Preferences Scale ${ }^{21}$ was used to evaluate the "amount of control patients want to assume in the process of making decisions" about the treatment of their diseases. It consists of five "cards" on a board, each illustrating a different role in decision making by means of a cartoon and short descriptive statement. The examiner asks the respondent to choose the preferred card, which is then covered up and cannot be chosen again; the examiner then asks the respondent to choose the preferred card from the remaining four cards. If the second preference is incongruent with the first (nonadjacent pairing, such as card A with card C), the test is explained again and is immediately readministered. In the event of a further incongruence, the test is not readministered, and a preference is not assigned. Administration of the test requires $\sim 5$ minutes. Six scores are possible based on the subject's two most preferred roles: active-active, active-collaborative, collaborative-active, collaborativepassive, passive-collaborative, and passive-passive. These scores are grouped as follows: active (active-active or active-collaborative), collaborative (collaborative-active or collaborative-passive), or passive (passive-collaborative or passive-passive). The scale has proven to be reliable (Cronbach's $\alpha=0.65)^{21}$

"Self-reported adherence" to psychiatric medication prescribed was assessed using the validated Spanish version of the eight-item self-report Morisky Medication Adherence Scale (MMAS-8). ${ }^{22,23}$ Questions are formulated to avoid a "yes-saying" bias (ie, the wording of item 5 is reversed to prevent the tendency to respond the same way to a series of questions regardless of their content). Response choices are "yes" or "no" for items 1 through 7 and item 8 has a five-point Likert response scale. Each "no" response is rated as 1 and each "yes" response is rated as 0 except for item 5, in which each "yes" response is rated as 1 and each "no" response is rated as 0 . For item 8 , the code $(0-4)$ has to be standardized by dividing the result by 4 to calculate a summated score. Total scores on the MMAS- 8 range from 0 to 8, with scores of 8 reflecting high adherence, 7 or 6 reflecting medium adherence, and $<6$ reflecting low adherence. The scale has proven to be reliable (Cronbach's $\alpha=0.83){ }^{22}$ Permission to use the scale was granted by Donald Morisky, the copyright holder of the instrument.

The Client Satisfaction Questionnaire (CSQ-8) ${ }^{24,25}$ was used to assess "global patient satisfaction", along a single dimension in the clinical setting. The CSQ-8 has eight question items (quality of service, kind of service, met needs, recommend to a friend, amount of help, deal with problems, overall satisfaction, and come back). Clients respond to those question items using a four-point Likert scale. Their responses are scored from 1 to 4 , and thus the possible total scores range from 8 to 32 . They can be used as such in data analysis or, as Larsen proposed, according to three levels: low (total score 8-20), medium (21-26), and high (27-32). The scale has proven to be reliable (Cronbach's $\alpha=0.87){ }^{24}$

\section{Statistical methods}

The data were analyzed using Statistical Package for Social Sciences (SPSS) software version 21 for Macintosh. ${ }^{26}$ Chisquare analyses were performed to contrast the differences among categorical variables. In order to analyze the pattern of relationships exhibited by the several categorical dependent variables considered, a multiple correspondences analysis (MCA) was carried out. MCA is an extension of the correspondences analysis that allows one to analyze the pattern of relationships exhibited by several categorical dependent variables. As such, it can also be seen as a generalization of principal component analysis when the variables to be analyzed are categorical rather than quantitative. ${ }^{27}$ The representation is usually done in a two-dimensional space that allows the observation of the proximity or remoteness of the categories. The closer the distance, the more frequent is the co-occurrence of these categories. Distances are measured in chi-square distances, being the model tested by this statistic.

\section{Results}

We recorded a high response rate of $77 \%$, resulting in a sample of 321 psychiatric outpatients. The 321 patients who agreed to participate in the study had a mean age of $48.4 \pm 15$ years (range $18-82$ years), and $75 \%$ were female. Concerning educational level, $15.3 \%$ of patients could only read and write, $47.4 \%$ had completed primary studies, $25.9 \%$ had completed secondary studies, and $11.5 \%$ had a university degree. The primary diagnoses of respondents were depressive disorders (57.3\%) and anxiety disorders (42.7\%). The average duration of treatment was $70 \pm 90$ months (range 2-600 months). The mean number of psychotropic drugs used was $2.2 \pm 1.5$ (range $0-5$ ). 

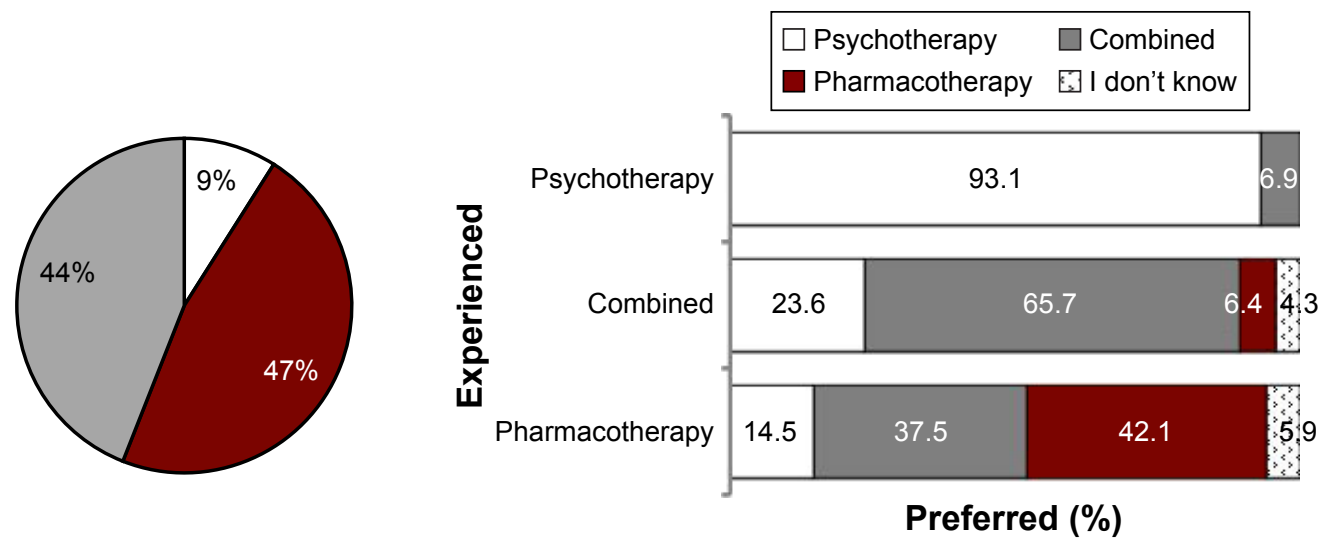

Figure I Self-reported treatment preferences according to experienced treatment modality.

Only 29 patients (9\%) self-reported being in exclusive treatment with psychotherapy, while $152(47.4 \%)$ reported they were on pharmacotherapy alone and $140(43.6 \%)$ with both combined treatment modalities. Combined treatment was the preferred option for the majority of patients $(47 \%$, 151 patients) followed by psychotherapy alone that was preferred by $22.7 \%$ ( 82 patients) of the sample. Concordance between experienced and preferred treatment alternative was $93.1 \%$ in psychotherapy modality, $42.1 \%$ in patients who received pharmacotherapy alone, and $65.7 \%$ in patients receiving both psychotherapy and pharmacotherapy combined. Figure 1 shows the distribution of preferred treatment options according to treatment experienced.

Of these 321 patients, only 108 (33.6\%) patients selfreported no decisional conflict since they scored 4 out of 4 ; 69 (21.5\%) scored 3; 72 (22.4\%) scored 2; 43 (13.4\%) scored 1 ; and $29(9 \%)$ scored 0 . Table 1 shows the percentage of psychiatric patients responding yes to each of the four-item SURE questions by treatment modality experienced. Neither sex nor educational level or patient diagnosis registered significant differences in decisional conflict prevalence. On the other hand, decisional conflict was more prevalent in patients with higher ages $(50.7 \pm 14.6$ vs $43.8 \pm 14.8$ years, $P=0.000$ ) and in those who underwent psychiatric treatment for a longer time (77.8 \pm 98 vs $53.7 \pm 79$ months, $P=0.027)$.
Decisional conflict registered significant differences between the different treatment categories experienced $37.9 \%$ in psychotherapy, $60.7 \%$ in combined treatment, and $77 \%$ in pharmacotherapy alone, $\chi^{2}=20.167, P=0.000$ ). Those patients who received their preferred treatment self-reported lower decisional conflict ( $62.3 \%$ vs $71.7 \%, \chi^{2}=3.143, P=0.049$ ).

Most outpatients (131 patients, $40.8 \%$ ) preferred shared decisional control, while 128 (39.9\%) preferred a passive approach and only $62(19.3 \%)$ an active decisional control. The most common preferred role was collaborative-passive $(27.4 \%)$ where doctor and patient share responsibility for deciding what treatment is best with the doctor making the final decision after considering the patient's opinion. The presence of decisional conflict did not influence the patients' preferences of participation in a significant way. The majority of patients under psychotherapy or combined treatment (43.3\% and $43.6 .1 \%$, respectively) expressed their preference for a collaborative approach, while patients under pharmacotherapy alone preferred a passive role (43.4\%). However, no significant differences were registered.

Concerning global satisfaction (CSQ-8, mean $=26.6 \pm 4.5$ ), distributions of responses indicated a very small proportion of dissatisfied or less satisfied patients. Only $7.5 \%$ of patients scored in the CSQ-8 "low" satisfaction category (total score 8-20), whereas $42.2 \%$ were satisfied (21-26)

Table I Percentage of psychiatric patients responding yes to each of the four-item SURE questions by treatment modality experienced

\begin{tabular}{|c|c|c|c|c|c|}
\hline Teatment modality & $\mathbf{n}$ & $\begin{array}{l}\text { Sure of } \\
\text { myself }\end{array}$ & $\begin{array}{l}\text { Understand } \\
\text { information* }\end{array}$ & $\begin{array}{l}\text { Risk-benefit } \\
\text { ratio** }\end{array}$ & Encouragement $* * *$ \\
\hline Psychotherapy alone & 29 & 79.3 & 82.8 & 75.9 & 86.2 \\
\hline Combined treatment & 140 & 75 & 69.3 & 57.1 & 72.9 \\
\hline Pharmacotherapy alone & 152 & 69.1 & 46.7 & 48.7 & 64.5 \\
\hline Global sample & 321 & 72.6 & 59.8 & 54.8 & 70.1 \\
\hline
\end{tabular}

Notes: Sure of myself: Do you feel sure about the best choice for you?; Understand information: Do you know the benefits and risks of each option?; Risk-benfit ratio: Are you clear about which benefits and risks matter most to you?; Encouragement: Do you have enough support and advice to make a choice?; $* P=0.000$, $* * P=0.020$, $* * * P=0.041$. Abbreviation: SURE, Sure of myself, Understand information, Risk-benefit ratio, Encouragement. 
and $50.3 \%$ very satisfied (27-32). Patients under psychotherapy self-reported the higher satisfaction (28.4 \pm 3.4$)$ followed by those under combined treatment (27.2 \pm 4.4$)$, and patients under pharmacotherapy alone were the less satisfied (25.6 \pm 4.6$)(P=0.000)$. Patients receiving preferred treatment self-reported higher satisfaction (concordant $=27.4 \pm 3.9$ vs nonconcordant $=25.8 \pm 4.7 ; P=0.000)$. Concerning decisional conflict, those patients without decisional conflict registered significantly higher satisfaction $(28.1 \pm 3.5 \mathrm{vs}$ 25.9 $\pm 4.7 ; P=0.000)$.

Regarding adherence to prescribed treatment, 37\% of the psychiatric outpatients self-reported a high level of adherence to their prescribed psychiatric drugs, while $33 \%$ self-reported medium adherence, and 30\% a low adherence (MMAS mean score 6.4 \pm 1.7 ). Analysis of variance (ANOVA) tests show significant differences in MMAS-8 scores only according to level of education (can read and

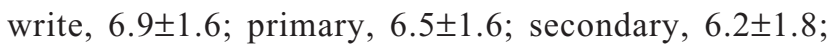
university, 5.9 $\pm 2 ; F[3,505]=2.96 ; P=0.035)$ and modality of treatment received (pharmacotherapy alone $=6.8 \pm 1.4 \mathrm{vs}$ combined treatment $=6.1 \pm 2.0, P=0.000$ ), but not in relation to patients' sex $(P=0.490)$, diagnoses $(P=0.400)$, decisional conflict $(P=0.4669)$, or concordance between received and preferred treatment $(P=0.644)$. Pearson's correlations carried out among MMAS- 8 score and age, time under treatment, and number of psychiatric drugs used did not register significant correlations (age, $P=0.278$; time under treatment, $P=0.345$; number of drugs used, $P=0.244$ ). Figure 2 shows psychiatric patients' decisional conflict, concordance between received and preferred treatment, preferences of participation, and adherence to prescribed treatment.

MCA performed showed a bidimensional solution. The first dimension with an eigenvalue of 1.723 reached a Cronbach's $\alpha$ of 0.525 and inertia value (variability index) of 0.345 , which corresponds to $56 \%$ of the explained variance. The second dimension with an eigenvalue of 1.282 reached a Cronbach's $\alpha$ of 0.275 and inertia value of 0.256 , corresponding to $43 \%$ of the explained variance. Table 2 shows the contribution of each variable to isolated dimensions. As can be observed, the variables that most contribute to dimension one are age and time under psychiatric treatment, while the variables that most contribute to dimension two are the satisfaction with health care received and the concordance between preferred and experienced modality of treatment.

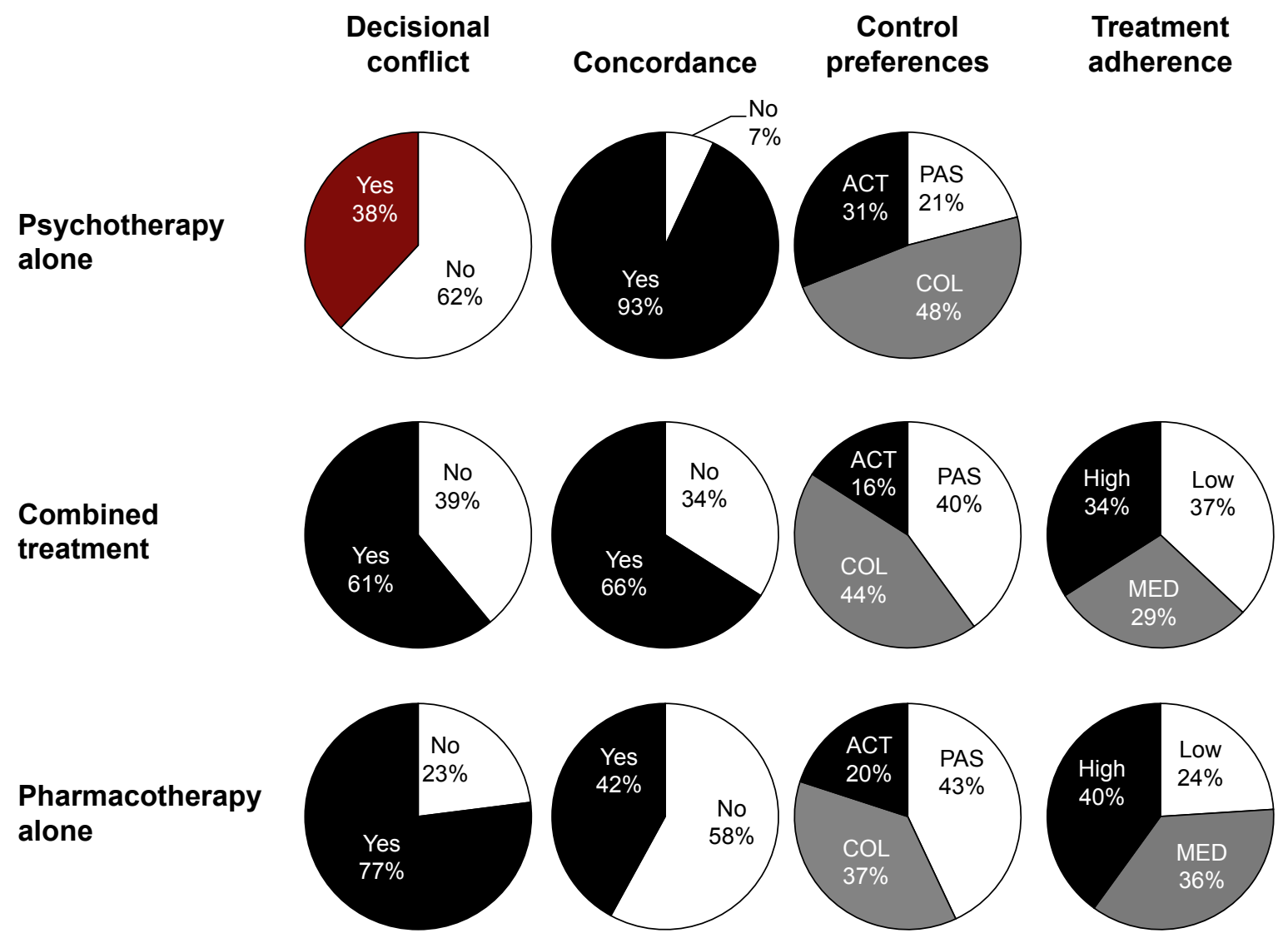

Figure 2 Psychiatric patients' decisional conflict, concordance between preferred and experienced treatment, preferences of participation, and adherence to prescribed treatment.

Abbreviations: ACT, active; COL, collaborative; PAS, passive; MED, medium. 
Table 2 Discrimination measures in dimensions of multiple correspondences analysis

\begin{tabular}{llll}
\hline Variables & \multicolumn{2}{l}{ Dimension } & Average \\
\cline { 2 - 3 } & $\mathbf{I}$ & $\mathbf{2}$ & \\
\hline Time under treatment & 0.417 & 0.074 & 0.245 \\
Diagnoses & 0.289 & 0.036 & 0.162 \\
Level of education & 0.323 & 0.040 & 0.182 \\
Experienced treatment & 0.307 & 0.276 & 0.292 \\
Agreement & 0.005 & 0.427 & 0.216 \\
Decisional conflict & 0.135 & 0.222 & 0.179 \\
Satisfaction & 0.035 & 0.486 & 0.260 \\
Preferred role & 0.199 & 0.081 & 0.140 \\
Age group & 0.468 & 0.016 & 0.242 \\
Total & 2.178 & 1.658 & 1.918 \\
\hline
\end{tabular}

Correspondences analysis of these data yields the graphical display (shown in Figure 3) that represents the two-dimensional graph generated from the estimated model showing the weight of the aforementioned variables and their contribution to explain the spread of the data. Two profiles of patients were evidenced when considering the relationships of decisional conflict with the variables of interest: a first profile characterized by patients without decisional conflict that received the treatment they preferred, mainly psychotherapy or combined treatment, that had been under psychiatric treatment for longer than 5 years, and self-reported high satisfaction with health care received; on the other hand, the

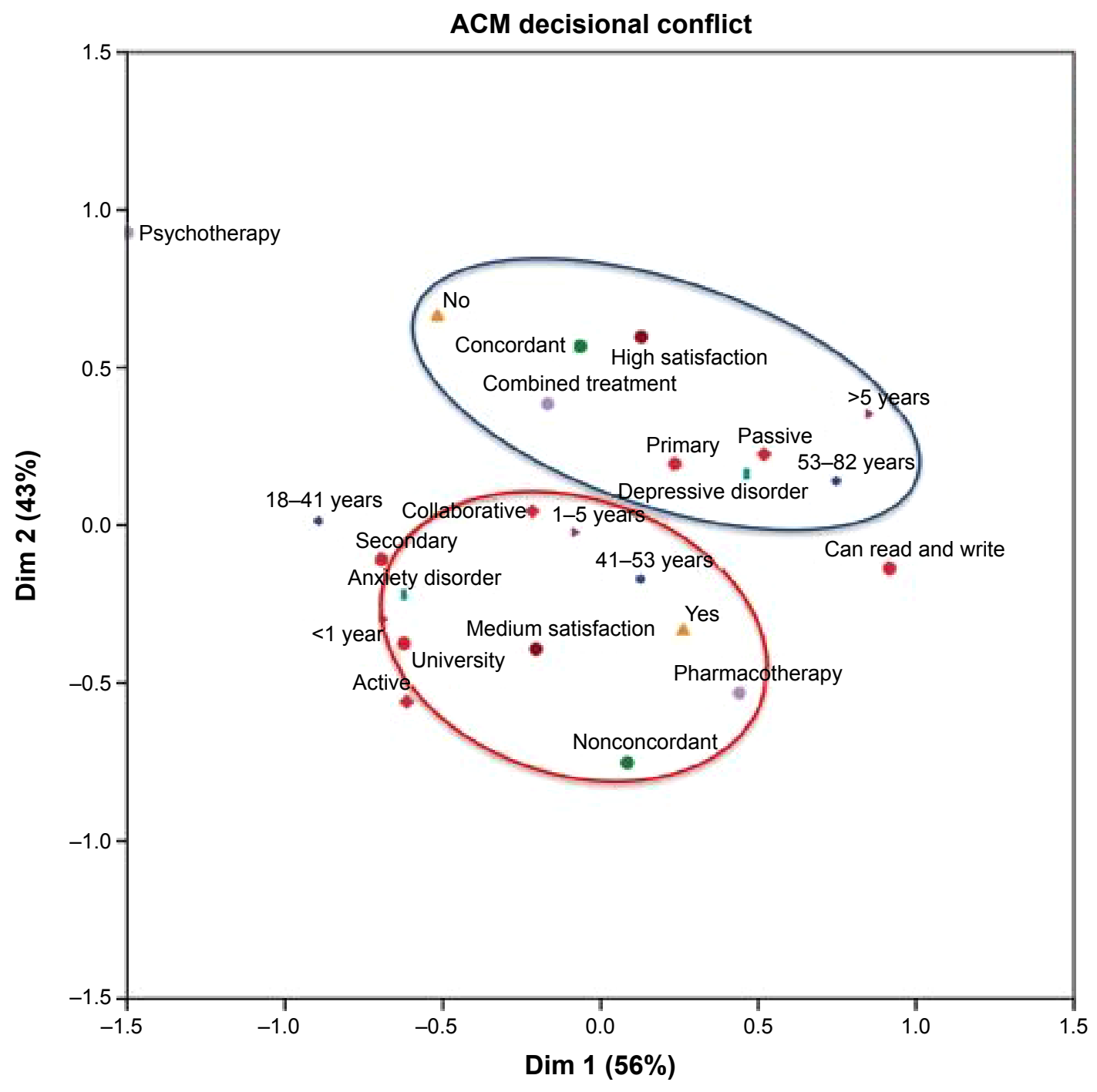

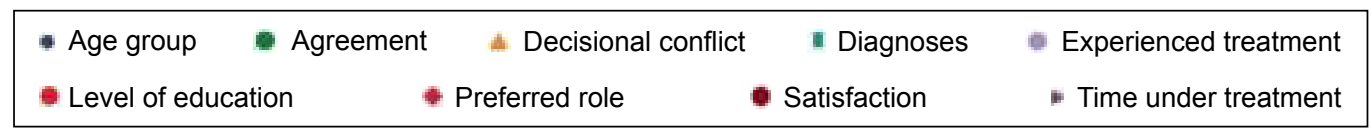

Figure 3 Dimensional representation of the multiple correspondences analysis of decisional conflict data. Abbreviations: Dim, dimension; ACM, multiple correspondence analysis. 
second profile included patients with decisional conflict that did not receive the treatment they preferred, that were treated with pharmacotherapy alone for a period of time between 1 and 5 years, and self-reported medium satisfaction with received health care.

\section{Discussion}

This study shows that there are high levels of decisional conflict in psychiatric outpatients with mood and anxiety disorders, revealing that involvement in psychiatric treatment decisions is an inherent part of decisional conflict, which supports our first working hypothesis (hypothesis 1 - see the section "Introduction"). Our results also show that age, treatment experienced, time under treatment, and concordance between preferred and experienced treatment are related to overall decisional conflict, and therefore any initiative to support decision taking by patients should be targeted to these variables. ${ }^{28}$

The higher rate of decisional conflict evidenced in older patients could reflect a more cautious approach of a more experienced service-user with his or her own preconceived opinions. However, other alternatives need to be taken into consideration, which were beyond the scope of our study but nevertheless deserve to be mentioned: Could it be that mental health professionals provide less information to elderly patients? Or is it possible that elderly patients have difficulties balancing risks and benefits? We should also consider the possibility that in patients with old age, younger mental health professionals could tend to offer less advice; finally, poor or absence of appropriate social supportive networks (ie, extended families, community support, etc, in Western societies) could account for this finding.

As would be expected, time under psychiatric treatment was associated with the presence of decisional conflict, and an inverse relationship between the two was evidenced. Thus, the shorter the time under psychiatric treatment, the higher the presence of decisional conflict, reflecting perhaps the difficulties of coming to terms with actual psychopharmacological treatment. On the other hand, decisional conflict decreases in patients who have received treatment for a longer time, highlighting patients' satisfaction with health care services and thus corroborating our second working hypothesis (hypothesis 2 - see the section "Introduction").

Concerning treatment preferred and experienced, a recent meta-analytic review of the literature on patient preferences for psychological versus pharmacological treatments for psychiatric disorders among adults has shown, after aggregation of patient preferences across diverse settings, a significant threefold preference for psychological treatment relative to medication. ${ }^{29}$ Although our knowledge of the impact of patient preferences on treatment course and outcome is limited, ${ }^{30}$ another meta-analysis examining the effect of treatment preference match on outcome across psychiatric conditions, a small but significant effect was found in favor of clients who received the treatment that they preferred. ${ }^{30}$ These findings support the idea that improving access to evidence-based psychological treatments is needed to connect more patients to their preferred treatment. ${ }^{29}$

Currently, limited research is available in clinical psychiatric practice on understanding how decision making preferences and processes impact the choices that are made by consumers, including service engagement and intervention outcomes. ${ }^{31}$ However, in general health care, the participation of patients in shared decision making has been shown to be associated with reduced decisional conflict, improved satisfaction, and improved communication between consumers and providers. ${ }^{32,33}$ Our results suggest that fostering a collaborative milieu is essential to reduce decisional conflict. Moreover, we also showed that the lesser the decisional conflict, the higher the patients' satisfaction.

The high degree of decisional conflict evidenced in our sample of psychiatric outpatients could be resolved by addressing through counseling or coaching the modifiable factors contributing to patients' uncertainty. Interventions should focus on the following: the possible lack of knowledge about options and potential outcomes of options; the unrealistic expectations or perceptions of the likelihood of positive outcomes, such as exaggerating or minimizing the chances of favorable outcomes; the unclear values, or the personal importance, or desirability of outcomes; the unclear perceptions of others, including opinions and practices; the social pressure to choose one option; the lack of support or mismatch between preferred and actual role in decision making; and the lack of skills/self-confidence. ${ }^{28}$ Psychiatrists may also reduce decisional conflict by meeting patients' desires for collaborative or active involvement in decision making. ${ }^{34}$ Future research should determine whether reducing decisional conflict leads to better outcomes of psychiatric patients.

This discussion would be unfinished if we did not mention the interesting fact that in our study, the presence of decisional conflict did not impact significantly adherence to treatment and patients' preferences of participation. These facts do not detract at all from the relevance of the 
aforementioned commentaries. New studies addressing this topic in different psychiatric disorders than anxiety and depression are needed.

\section{Limitations}

There are several limitations related to the methodology of this study that need to be considered. First, although a high rate of participation was recorded, results registered may be affected by a selection bias. Specifically, there may be differences between individuals who agreed to participate in the study and those who did not. Second, the study included a convenience sample of consecutive psychiatric outpatients diagnosed of depressive and anxiety disorders attending community mental health centers and therefore is representative only of these kinds of patients at this health care setting. Finally, the study relied entirely on self-report measures which carry a potential risk of misstatement or response biases.

\section{Conclusion}

Our study highlights the crude reality in which psychiatrists currently live and in which they have to deal with decisional conflict in their daily clinical practice with psychiatric outpatients with affective and anxious disorders. Age, treatment experienced, and concordance between treatments preferred and experienced are relevant factors when decisional conflict is present. It is fundamental that psychiatrists and other mental health professionals endeavor to provide patients with tailored information and skills about illnesses and treatments that may facilitate patients' involvement in the decision making process and reduce the decisional conflict reported by psychiatric patients.

\section{Acknowledgments}

This paper is part of a wider research project (PI10/00955) supported by the Instituto de Salud Carlos III and the European Union - Fondo Europeo de Desarrollo Regional. This study shares parts of its methodology and evaluation methods with previous studies as referenced. ${ }^{1,6,23,35-43}$

\section{Disclosure}

The authors report no conflicts of interest in this work.

\section{References}

1. De las Cuevas C, Peñate W. Preferences for participation in shared decision making of psychiatric outpatients with affective disorders. OJPsych. 2014;4:16-23.

2. Fraenkel L, McGraw S. Participation in medical decision-making: the patients' perspective. Med Decis Making. 2007;27(5):533-538.
3. Joosten EA, DeFuentes-Merillas L, de Weert GH, Sensky T, van der Staak CP, de Jong CA. Systematic review of the effects of shared decision-making on patient satisfaction, treatment adherence, and health status. Psychother Psychosom. 2008;77:219-226.

4. Funk LM. Who wants to be involved? Decision-making preferences among residents of long-term care facilities. Can J Aging. 2004;23: $47-58$.

5. Levinson W, Kao A, Kuby A, Thisted RA. Not all patients want to participate in decision making. A national study of public preferences. J Gen Intern Med. 2005;20:531-535.

6. De las Cuevas C, Peñate W, Perestelo-Pérez L, Serrano-Aguilar P. Shared decision-making in psychiatric practice and the primary care setting is by no means the same, according to SDM-Q-9. Neuropsychiatr Dis Treat. 2013;9:1045-1052.

7. Groopman JE, Hartzband P. Your Medical Mind: How to Decide What Is Right for You. New York: Penguin Press; 2011.

8. Kessler RC, Berglund P, Demler O, Jin R, Merikangas KR, Walters EE. Lifetime prevalence and age-of-onset distributions of DSM-IV disorders in the National Comorbidity Survey Replication. Arch Gen Psychiatry. 2005;62(6):593-602.

9. Gorman JM. Comorbid depression and anxiety spectrum disorders. Depress Anxiety. 1996-1997;4:160-168.

10. Hunt C, Issakidis C, Andrews G. DSM-IV generalized anxiety disorder in the Australian National Survey of Mental Health and Well-Being. Psychol Med. 2002;32:649-659.

11. Pollack MH. Comorbid anxiety and depression. J Clin Psychiatry. 2005;66(8):22-29.

12. Barlow DH, Allen LB, Choate ML. Toward a unified treatment for emotional disorders. Behav Ther. 2004;35:205-230.

13. McEvoy PM, Nathan P, Norton PJ. Efficacy of transdiagnostic treatments: a review of published outcome studies and future research directions. J Cogn Psychother. 2009;23:20-33.

14. Loh A, Leonhart R, Wills CE, Simon D, Härter M. The impact of patient participation on adherence and clinical outcome in primary care of depression. Patient Educ Couns. 2007;65(1):69-78.

15. Huhn M, Tardy M, Spineli LM, et al. Efficacy of pharmacotherapy and psychotherapy for adult psychiatric disorders: a systematic overview of meta-analyses. JAMA Psychiatry. 2014;71(6):706-715.

16. Cuijpers P, Sijbrandij M, Koole SL, Andersson G, Beekman AT, Reynolds CF. The efficacy of psychotherapy and pharmacotherapy in treating depressive and anxiety disorders: a meta-analysis of direct comparisons. World Psychiatry. 2013;12(2):137-148.

17. Amick HR, Gartlehner G, Gaynes BN, et al. Comparative benefits and harms of second generation antidepressants and cognitive behavioral therapies in initial treatment of major depressive disorder: systematic review and meta-analysis. BMJ. 2015;8(351):h6019.

18. O'Connor A. Decisional conflict. In: McFarland GK, McFarlane EA, editors. Nursing Diagnosis and Intervention, 3rd ed. Toronto, Canada: Mosby; 1997:486-496.

19. World Health Organization. The ICD-10 Classification of Mental and Behavioural Disorders. Clinical Descriptions and Diagnostic Guidelines. Geneva: World Health Organization; 1992.

20. Légaré F, Kearing S, Clay K, et al. Are you SURE? Assessing patient decisional conflict with a 4 -item screening test. Can Fam Physician. 56(8); $\mathrm{e} 308-\mathrm{e} 314$.

21. Degner LF, Sloan JA, Venkatesh P. The Control Preferences Scale. Can J Nurs Res. 1997;29:21-43.

22. Morisky DE, Ang A, Krousel-Wood M, Ward H. Predictive validity of a medication adherence measure for hypertension control. J Clin Hypertens. 2008;10(5):348-354.

23. De las Cuevas C, Peñate W. Psychometric properties of the eight-item Morisky Medication Adherence Scale (MMAS-8) in psychiatric outpatient setting. Int J Clin Hlth Psyc. 2015;15:121-129.

24. Larsen DL, Attkisson CC, Hargreaves WA, Nguyen TD. Assessment of client/patient satisfaction: development of a general scale. Eval Program Plann. 1979;2:197-207. 
25. Echeburúa E, Corral P. Manual de violencia familiar [Manual of domestic violence]. Madrid: Siglo XXI;1998:50-51.

26. IBM Corporation. IBM SPSS Statistics for Macintosh, Version 21.0. Armonk, NY: IBM Corporation; 2012.

27. Abdi H, Valentin D. Multiple correspondence analysis. In: Neil Salkind, editor. Encyclopedia of Measurement and Statistics. Thousand Oaks, CA: Sage; 2007.

28. O'Connor AM, Jacobsen MJ. Decisional conflict: supporting people experiencing uncertainty about options affecting their health. Ottawa Hospital Research Institute; 2006. Available from: http://careinaging. duke.edu/longtermcare/dynamic/resources/1027/Decisional_Conflict. pdf. Accessed May 27, 2016.

29. McHugh RK, Whitton SW, Peckham AD, Welge JA, Otto MW. Patient preference for psychological vs pharmacologic treatment of psychiatric disorders: a meta-analytic review. J Clin Psychiatry. 2013;74(6): 595-602.

30. Winter SE, Barber JP. Should treatment for depression be based more on patient preference? Patient Prefer Adherence. 2013;9(7):1047-1057.

31. Swift JK, Callahan JL. The impact of client treatment preferences on outcome: a meta-analysis. J Clin Psychology. 2009;65(4):368-381.

32. Cooper LA. At the center of the decision-making in mental health services and interventions research: patients, clinicians, or relationships? Clin Psychol Sci Prac. 2006;13:26-29.

33. O'Connor AM, Bennett CL, Stacey D, et al. Decision aids for people facing health treatment or screening decisions. Cochrane Database Syst Rev. 2009;(3):CD001431.

34. Thistlethwaite J, Evans R, Tie RN, Heal C. Shared decisión making and decision aids: a literature review. Aust Fam Physician. 2006;35(7): $537-540$.

35. De las Cuevas C, Rivero A, Perestelo-Pérez L, González M, Pérez J, Peñate W. Psychiatric patients' attitudes towards concordance and shared decision making. Patient Educ Couns. 2011;85:e245-e250.
36. De las Cuevas C, Rivero-Santana A, Perestelo-PérezL, Serrano-Aguilar P. Attitudes toward concordance in psychiatry: a comparative, crosssectional study of psychiatric patients and mental health professionals. BMC Psychiatry. 2012;12:53.

37. De las Cuevas C, Peñate W, Perestelo-Pérez L, Serrano-Aguilar P. Shared decision-making in psychiatric practice and the primary care setting is by no means the same, according to SDM-Q-9. Neuropsychiatr Dis Treat. 2013;9:1045-1052.

38. De las Cuevas C, Peñate W, Sanz EJ. Psychiatric outpatients' selfreported adherence versus psychiatrists' impressions on adherence in affective disorders. Hum Psychopharmacol. 2013;28(2):142-150.

39. De las Cuevas C, Peñate W, De Rivera L. Psychiatric patients' preferences and experiences in clinical decision-making: examining concordance and correlates of patients' preferences. Patient Educ Couns. 2014;96(2):222-228.

40. De las Cuevas C, Peñate W. To what extent psychiatric patients perceive involvement in decision-making about their mental health care? Relationships with socio-demographic, clinical and psychological variables. Acta Neurpsychiatr. 2014;26(6):372-381.

41. De las Cuevas C, Peñate W, De Rivera L. To what extent is treatment adherence of psychiatric patients influenced by their participation in shared decision-making? Patient Prefer Adherence. 2014;8: $1547-1553$.

42. De las Cuevas C, Perestelo-Perez L, Rivero-Santana A, Cebolla-Martí A, Scholl I, Härter M. Validation of the Spanish version of the 9-item Shared Decision Making Questionnaire. Health Expect. 2015;18(6): 2143-2153.

43. De las Cuevas $C$, Peñate $\mathrm{W}$. Explaining pharmacophobia and pharmacophilia in psychiatric patients: relationship with treatment adherence. Hum Psychopharmacol. 2015;30(5):377-383.
Patient Preference and Adherence

\section{Publish your work in this journal}

Patient Preference and Adherence is an international, peer-reviewed, open access journal that focuses on the growing importance of patient preference and adherence throughout the therapeutic continuum. Patient satisfaction, acceptability, quality of life, compliance, persistence and their role in developing new therapeutic modalities and compounds to optimize

\section{Dovepress}

clinical outcomes for existing disease states are major areas of interest for the journal. This journal has been accepted for indexing on PubMed Central. The manuscript management system is completely online and includes a very quick and fair peer-review system, which is all easy to use. Visit http://www dovepress.com/testimonials.php to read real quotes from published authors. 